\title{
An Artificial Neural Network (ANN)-Based Learning Agent for Classifying Learning Styles in Self-Regulated Smart Learning Environment
}

\author{
https://doi.org/10.3991/ijet.v16i18.24251 \\ Yusufu Gambo $\left.{ }^{(}\right)$, Muhammad Zeeshan Shakir \\ University of the West of Scotland, Paisley, United Kingdom \\ b00320837estudentmail.uws.ac.uk
}

\begin{abstract}
The increasing development in smart and mobile technologies transforms learning environments into smart learning environments. Students process information and learn in different ways, and this can affect the teaching and learning process. To provide a system capable of adapting learning contents based on students' learning behavior in a learning environment, the automated classification of the learners' learning patterns offers a concrete means for teachers to personalize students' learning. Previously, this research proposed a model of a self-regulated smart learning environment called the metacognitive smart learning environment model (MSLEM). The model identified five metacognitive skills-goal settings (GS), help-seeking (HS), task strategies (TS), time-management (TM), and self-evaluation (SE) that are critical for online learning success. Based on these skills, this paper develops a learning agent to classify students' learning styles using artificial neural networks (ANN), which mapped to FelderSilverman Learning Style Model (FSLSM) as the expected outputs. The receiver operating characteristic (ROC) curve was used to determine the consistency of classification data, and positive results were obtained with an average accuracy of $93 \%$. The data from the students were grouped into six training and testing, each with a different splitting ratio and different training accuracy values for the various percentages of Felder-Silverman Learning Style dimensions.
\end{abstract}

Keywords-Self-regulated learning, smart learning environment, personalized learning, learning styles, artificial neural network

\section{Introduction}

Every student learns and processes information differently, mainly due to their behavioral or cultural differences $[1,2,3]$. Some prefer text or audio, while others prefer video, exercise, collaboration, inquiry, demonstration, etc. These learning differences can be classified and develop in an online learning environment to support students' learning needs $[4,5]$. Furthermore, with the current Covid-19 pandemic that has disrupted many educational institutions, there is a need to provide a personalized learning system based on students' learning styles that can support remote and isolated learners and mitigate challenges caused by disruptions in the learning process. Classifying the 
learning style is essential because it enhances learning efficiency, increases motivation, and minimizes learning time. This process also allows educational institutions to provide learning materials to the learning styles of their students. From a theoretical point of view, learning styles are represented in models of learning styles [5, 6]. In the past two decades, a great deal of interest in learning styles has contributed to the explosion of learning models. Several models have also been proposed based on an interpretation of the various features of the learners. These include the Kolb model [7], the Gardner model [8], the Felder and Silverman model [9], and the Biggs model [10]. The critical challenge is how to classify the learning style of the student based on these models.

There are two major approaches to classifying learning styles that have been pursued in literature: the traditional approach and the automated approach. The traditional method is to classify learning preferences by a questionnaire that learners are asked to complete. However, this approach poses several drawbacks that are primarily linked to the student's lack of enthusiasm to complete the questionnaire and knowledge of their learning interests. Automatic methods have since been implemented to overcome the shortcomings of the traditional solution. These automated methods consist of collecting experience from learners' engagement with a learning environment, which can be errorfree. Several studies have used various learning styles and artificial intelligence methods to process data from the learning environment to classify students' learning styles. A wide range of methods for the automated classification of learning styles have been suggested, such as the Bayesian networks method, the decision tree techniques, and neural networks [11-15]. Although these classification methods yield substantial outcomes, their efficiency differs from one method to another [11, 13, 14, 16].

Previously, this research proposed a model of a self-regulated smart learning environment called the MSLEM-metacognitive smart learning environment model [17]. The model consists of six connected modules, i.e., cognitive detection, learning contents management, adaptive assessment, inference engine, metacognitive and intervention engine; it identified five metacognitive skills-goal settings (GS), help-seeking (HS), task strategies (TS), time-management (TM), and self-evaluation (SE) as the critical success skills for a self-regulated smart learning environment. A learning agent needs to be developed and integrated into the model's inference engine module to provide a system capable of personalizing learning contents to students based on their behavior in a learning environment [17].

This paper describes an approach for mapping students' actions (metacognitive skills) in a self-regulated smart learning environment into learning styles. The method used an artificial neural network (ANN), a computational model for classification, and has proven to produce an accurate classification. The learning agent development can be used in the inference engine of the MSLEM for an intelligent decision based on students' learning behavior for supporting the development of a self-regulated smart learning environment. 


\subsection{Classification}

Data mining is used to derive valuable information from data by classifying patterns. It is mostly used in computing areas such as pattern recognition and database management. A model is often employed to classify well-grounded data, and it's also used to provide a mathematical model for larger datasets. In machine learning, a classification problem is when the input data is grouped into one of the predefined labeled groups. Predicting Yes or No, True or False, for example, comes into the definition of Binary classification when the number of outputs is reduced to two labels. Similarly, the performance of multiple classes, such as classifying various age ranges, is referred to as a multiclass classification challenge. Classification problems are among the most widely used or specified types of machine problems used in various applications $[11,12,18]$.

\subsection{Classification Procedures}

There are two kinds of classifications: supervised classifications and unsupervised classifications. Supervised processes are classified as those that allow the user to pick a testing dataset, run the classification algorithm on it, and then generate a model that can calculate the performance and accuracy of the test dataset. On the other hand, unsupervised classification results are based on software interpretation of the components, with no sample groups defined by the consumer. Data processing methods are used to decide which objects are associated, and they must belong to actual features to classify them [18]. This study focuses on supervised classification, consisting of the following steps $[11,12,19]$.

Data Collection and Feature Extraction. The pre-processing procedure is the first step in compiling raw data. It is preferable to discard sample rows with no values and attribute columns with no results in function extraction.

Sampling. Following the extraction of features from raw data, the sample must be arbitrarily divided into testing and evaluation datasets. The training dataset would be used to put the model through its paces. The test dataset will then be used to assess the final model's results.

Normalization. The normalization function technique is used to compare different attributes, particularly when measured on different scales, and it is a requirement for machine learning algorithms

Validation. Validation is one of the most useful techniques for bringing various feature selection, dimensionality reduction, and learning algorithms to the test.

\subsection{Classification Models}

For an algorithm to have some value, there must be a clear input or outcomes that it produces. When running, the algorithm performs features that process a training dataset that incorporates many attributes. The algorithm is designed to search for relationships between characteristics that could be used to predict the result. Many learning algo- 
Paper-An Artificial Neural Network (ANN)-Based Learning Agent for Classifying Learning...

rithms are used, such as the decision tree, artificial neural network, Naïve Bayes, Random forest, etc. $[11,12,18]$. These models can be used to classify outputs based on the combination of inputs.

\section{$2 \quad$ Related Works}

Various classification approaches have been used in literature to automatically classify learning styles of distinct learning patterns [11-15]. These methods use the data obtained from the learner's experiences with the system. In this method, the identification is made by an artificial intelligence classification algorithm. The learner's actions are taken as input to the algorithm, and the learners' learning style expectations were returned as output. Besides, in the automated classification of learning types, various learning activity facets must be considered when developing a detection strategy. Also, these aspects are required in an experiment to achieve a level of accuracy.

Bajaj and Sharma [12] considered smart education with artificial intelligence based on learning style. The aim is to customize learning contents and learning paths for a student. This aid's in minimizing disorientation and cognitive overload problems. The authors compared the simulated experiment with Felder-Silverman and Kolb's learning styles, multi-layer perceptron, and decision tree. The results of the comparison show that Felder Silverman/Multi-layer Perceptron has the highest correct classification. They suggested that the framework be deployed as a cloud-based service to provide adaptive learning to students worldwide. Melesko and Kurilovas [15] presented a framework for customizing learning and a model for personalized multi-agent intelligent learning systems for engineering courses based on student's learning styles and other personal characteristics. This model used Felder Silverman's learning style and ontologies-based personalized recommender system to provide an individualized recommendation to students. While the framework is still at the experiment level, it can be extended and enriched to provide and support students' online process.

Kolekar et al. [13] used the Fuzzy C Means (FCM) algorithm to organize the captured learning behavioral data into the FSLSM groups. In this algorithm, the contents and interfaces appropriate for that category are given to each learner category. The Gravitational Search-based Back Propagation Neural Network (GSBPNN) algorithm is used to classify learner learning styles in real-time, providing a good prediction accuracy. Azzi et al. [11] used an automated identification of learners' learning styles in elearning to personalized learning materials. The student's learning activity is captured in several ways, usually in various courses relating to a single subject. Web usage mining is used to capture learning patterns, and then learning types are mapped to the Felder-Silverman Learning Style Model (FSLSM) groups. Fuzzy C means (FCM) algorithm is used to organize collected learning behavioral data into FSLSM groups. The experimental findings demonstrated the method's success since the data obtained are gathered from the learners' learning activities referring to multiple courses.

Kose [14] proposed an ANN-based agent to improve the learning experience. The system consists of an architecture based on ANN to determine students' learning styles and grades before feeding their interface with the course's related content. The ANN 
architecture is primarily provided with responses given for a specially designed multiple intelligence test. This information is also combined with other data, such as exam grades and points offered for each participant by the course instructor. The survey assessment findings indicate that the students who took part in the experimental group are positive about the conducted educational process and the software system. Many students believe that the course content of the program was sufficient for their interests and motivation. While this system could support students based on the evaluation parameters, it can be extended to consider personal characteristics and other evaluation parameters to increase their applicability and generalization.

Similarly, Zakrzewski et al. [16] used an artificial neural network to detect individual differences in metacognitive monitoring. The authors trained the network to recognize individuals as younger or older, and their hidden behavior was analyzed to detect data trends that separated younger participants from older ones. The analysis of the hidden unit interactions showed that the network could accurately identify three separate groups of participants.

The related works show that ANN has been extensively used to classify learning styles based on students' behavioral data; however, it's still rarely used to model metacognitive skills to classify learning styles to support learning personalization in an online learning environment. Furthermore, FSLSM has become the most commonly used learning style model to support learning style classification. Thus, this paper adopted ANN and FSLM to model, train, and test the student learning style classification.

\subsection{Artificial Neural Network}

An artificial neural network (ANN) is composed of a cell linked to a system; it is a computational model based on the brain's biological neural structure; and every link has a numeric weight, which infers the importance of that link [20-22]. The network consists of an input layer, a hidden layer, and an output layer. In the input layer, the number of neurons is the same as the number of input signals for the process, provided that there is no bias signal. If bias is added at the input layer, it is treated as a new neuron to the input layer. There may be more than one hidden layer in ANN, but using a single hidden layer is a universal approximator [21, 22]. Every neuron in the hidden layer receives a signal from each neuron of the input layer. It computes it by combining them and applying an activation function [20, 23], and the final layer is the output layer.

An ANN learns by internal changes so that it adapts its' behavior to the environment. When an external agent is involved in the learning process, it is called supervised learning. This type of network is called feed-forward in multi-layer perceptron since it has no recurrent feed-back layers and is the most popular to deal with in the multi-input and multi-output case. In the multi-layered network, the learning is accomplished with the back-propagation algorithm, a supervised learning algorithm based on the error function that needs to be minimized over the weight space [24, 25]. This process works in two steps. The first step is called the training process, where the network is initializing with some small random values of weights. The essence is to find a set of weight values that minimize the global error of the system. 
In contrast, the second step is called generalization. The system has already learned an internal representation of the earlier presented patterns and can classify novel patterns presented as input. ANN offers many advantages: Neuro-biological, Non-linearity, Adaptive process, Input-output mapping, fault-tolerant, uniform design, analysis, etc. It appears in most learning style classification [13, 15, 25].

\subsection{Felder and Silverman Learning Style Model}

Several learning styles model has been discussed in literature such as the Kolb, Myers \& McCaulley, Felder \& Silverman (FSLSM). However, FSLSM is the most common and widely used in literature because of its' flexibility and ability to capture different learning style dimensions [11-14]. The FSLS [9] was developed to assist both students and facilitators. It can classify an individual student's learning style to provide them with learning resources that fit them. The model consists of eight sub-dimensions under four dimensions and are discussed as follows:

- Perception: This explains the kind of knowledge a student perceives ideally. It is categorized into Sensing and Intuitive: Sensing learners believe in facts and rely on tried and true methods when solving a problem. In contrast, intuitive learners are more interested in novel and creative ideas. They always look for new ways of addressing old issues, which are more drawn to hypothetical scenarios and abstract things.

- Input: External information is perceived effectively through input via sensory channels. Visual user best learns by what they see, while verbal learners learn by what they hear.

- Processing: This provides ways by which learners process information which can either be Active or Reflective: The active users are more interested in in-class activities and apply the material to bring out things by learning them actively, whereas reflective users are buried with group activities and process better they think individually.

- Understanding: How best does the student proceed towards understanding which is divided into sequential or Global: Sequential user learns step by step, whereas Global one learns by grasping a large picture in mind and using a Holistic thought process.

Table 1 shows the Felder \& Silverman Learning Model (FSLM) and the associated learning objects $[9,11]$.

Table 1. Felder \& Silverman Learning Model Sub-Dimensions

\begin{tabular}{|l|c|}
\hline \multicolumn{1}{|c|}{ FSLSM Sub-Dimensions } & Learning Objects \\
\hline Active & Videos, PPTs, Demo, Exercise, Assignments \\
\hline Reflective & PDFs, PPTs, Videos, Announcements, References \\
\hline Sensing & Examples, PDFs, Videos, Practical Material \\
\hline Intuitive & PDFs, PPTs, Videos, Forum, Topic, List, References \\
\hline Visual & Images, Charts, Videos, References \\
\hline Verbal & PDFs, Videos, Email, Announcements \\
\hline Sequential & Exercise, References, Assignments, Sequential \\
\hline
\end{tabular}




\section{Our Proposed Approach}

The proposed approach to provide personalized learning content in a self-regulated smart learning environment is to develop a learning agent that can be integrated into the inference engine module of the MSLEM [17]. However, the reliability of adaptive depends on the precision of the learning style classification. Indeed, this accuracy relies on the availability of data as the basis for classifying learning styles.

We used three approaches for developing the learning agent to classify learning styles. The first was modeling ANN-based learning agents based on the inputs and expected outputs. The second step is getting available data for the experiment. The third stage is to train and evaluate the model through an experiment to classify the learning style and make appropriate recommendations based on students' actions in the selfregulated smart learning environment, as shown in Fig. 1. The combinations of goalsetting (GS), task strategy (TS), help-seeking (HS), time management (TM), and selfevaluation (SE) determine each of the learning styles to recommended personalized learning resources.

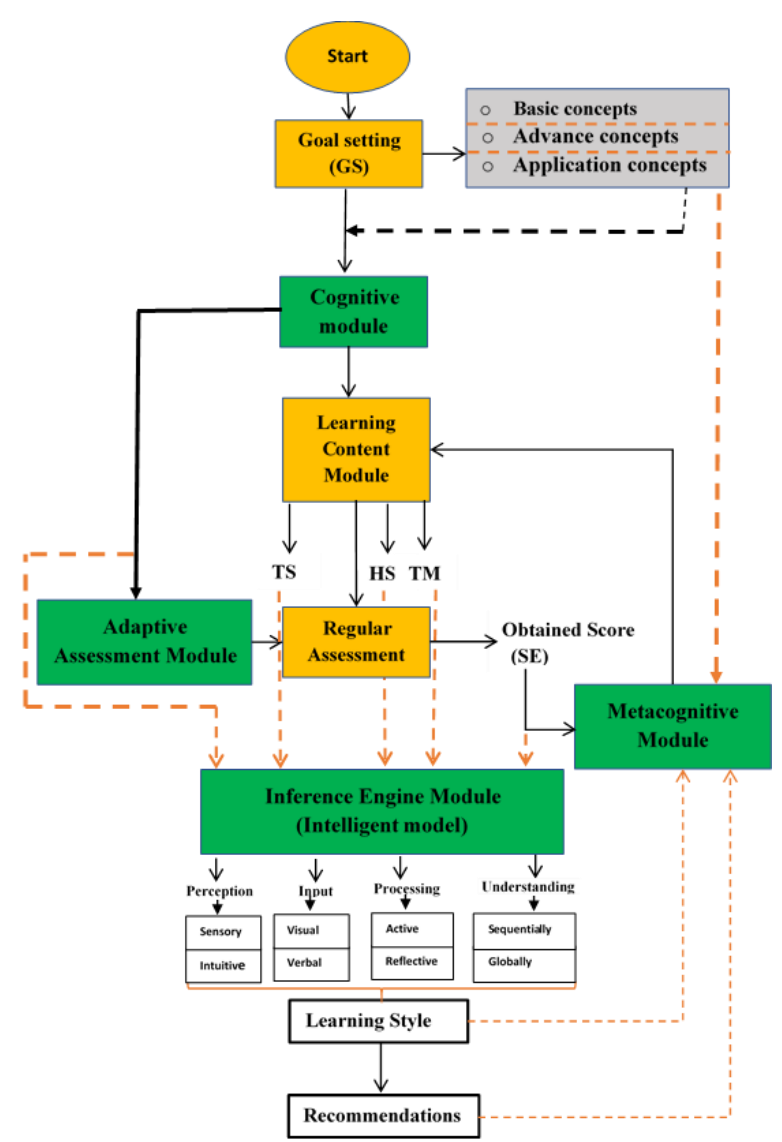

Fig. 1. The Overall data Flow for the Proposed Approach 


\section{$4 \quad$ Modeling ANN-Based Learning Agent}

The ANN takes five inputs, a simulated student's learning behavior, and four outputs, which indicates the student's learning style based on FSLSM [9]. The ANN is trained with the help of this input-output paired dataset. After training, the ANN can classify the four output values based on their learning behavior. The ANN learning agent consists of the following layers:

\subsection{Input Layer}

This layer contains the number of input signals to be used while classifying the output for a particular process. A total of five inputs are used to classifying the learning style of a student. A detailed description of these five inputs are:

- Goal Setting (GS): A predefined goal is required to be selected by students, like how they are interested in achieving: basic concepts, advanced concepts, or application concepts. Each of these goals has an expected score, i.e., basic (50\%), advance $(60 \%)$, and application (70\%) and above), which is to be used as one of input into ANN.

- Task Strategy (TS): The student will have access to learning content based on the FSLM sub-dimensions, i.e., text, pdf, video, outline, exercises, and examples updated on learning contents management module. The frequency of using each is captured to determine the specific learning style

- Help-Seeking (HS): While in the learning environment, there is a discussion forum where students can interact with peers or facilitators for help, announcement, or clarification, and the frequency of using the forum is also captured.

- Time-Management (TM): Here, the time spent learning the course content and taking the assessment is captured.

- Self-Evaluation (SE): A student must appear for an objective type assessment generated by the adaptive assessment module. The evaluation assessment is developed with the help of the inference engine based on the goal selected, i.e., basic conceptssimple question, advance concepts-advance question, or application concept-practical question. Students can take the assessment as many times. The score obtained in the assessment becomes the student's current knowledge. It is displayed in the metacognitive module, which will be available for both the individual student and facilitator, as shown in Fig.1.

Based on Table 1, and following the methodology of El-Bishouty [27] for mapping learning objects to learning styles, GS is intuitive since student selects a learning goal based on the insights and knowledge of their understanding of the subject. TS is the learning object in the learning content management module. However, the frequency of using specific content, i.e., audio, pdf, video, outline presentation, etc., is captured. Thus, TS uses the learning style to determine a particular learning style. HS is a discussion forum object that falls under intuitive, verbal, and reflective. A student can use the discussion forum to seek help, read comments, announcements, and send comments. 
TM is an essential component in this context. The amount of time student spent on learning and assessment have effects on their achievement and learning style. A student who wants a summary of the subject might use a presentation not to waste much time reading through the learning contents. Thus, TM is measured across the learning styles to determine student-specific learning styles. Lastly, SE falls under both active and global. Students can use self-assessment to gauge their understanding of a topic or as a reflective exercise, which can be input into learning style. The mapping of the five skills based on FSLM is shown in Table 2.

Table 2. Mapping of metacognitive skills (Inputs) to FSLM Sub-Dimensions

\begin{tabular}{|l|c|c|c|c|c|c|c|c|}
\hline $\begin{array}{c}\text { Learning Object/ } \\
\text { Learning Style }\end{array}$ & Sensory & Intuitive & Visual & Verbal & Active & Reflective & Sequence & Global \\
\hline GS & & $\mathrm{X}$ & & & & & & \\
\hline TS & $\mathrm{X}$ & $\mathrm{X}$ & $\mathrm{x}$ & $\mathrm{x}$ & $\mathrm{X}$ & $\mathrm{x}$ & $\mathrm{X}$ & $\mathrm{x}$ \\
\hline HS & & $\mathrm{X}$ & & $\mathrm{x}$ & & $\mathrm{x}$ & & \\
\hline TM & $\mathrm{X}$ & $\mathrm{X}$ & $\mathrm{x}$ & $\mathrm{x}$ & $\mathrm{X}$ & $\mathrm{x}$ & $\mathrm{X}$ & $\mathrm{x}$ \\
\hline SE & & & & & $\mathrm{X}$ & & & $\mathrm{x}$ \\
\hline
\end{tabular}

\subsection{Output Layer}

The expected outputs to the input combinations for each of the FSLM dimensions [9] are based on the learning objects in Table 1 and mapping categorization in Table 2, as shown in Table 3.

Table 3. Mapping of metacognitive skills (Inputs) to FSLM Sub-Dimensions

\begin{tabular}{|l|c|c|}
\hline Expected Output & Input Combinations & FSLM Dimensions \\
\hline Y1 & GS, HS, TM, TS & Perception (Sensory/Intuitive) \\
\hline Y2 & HS, TS, TM & Input (Visual/Verbal) \\
\hline Y3 & HS, TS, TM, SE & Processing (Active/Reflective) \\
\hline Y4 & TS, TM, SE & Understanding (Sequential/Global) \\
\hline
\end{tabular}

\subsection{Data Set}

The data set was generated by stimulating students' learning behavior based on the five inputs-GS, HS, TS, TM, and SE to evaluate the proposed approach. This is due to the lack of homogenous data to build learning sequences to map to the learning styles dimension. We set arbitrary weight values to each input and normalized them to generate the input data sets. The normalized values are used to randomly generated 1000 data sets using Microsoft Excel. We considered that each student has a particular learning style, preference, and behavior for the outputs. Based on the input combinations for each FSLM in Table 3, we computed each expected outcome's value and then used Microsoft Excel to generate 1000 data sets. The sample input-output pairs of the simulated data for the experiment are attached as supplementary material. 


\subsection{Training Phase}

We used a multi-layer perception network to train the current data using MATLAB R2016a with a three-layered ANN structure of 5-4-4. The first layer contains 5 input neurons; the second layer hidden layer contains 4 processing units connected to 4 outputs in the third layer. Learning rate and momentum term have a great influence on the training of the ANN because a very small value may result in slower learning of ANN. In contrast, a large value may result in unstable learning, so these values should be chosen carefully. Here, these values are selected by trial and error, starting from a small value. The learning rate is set to 0.02 , and momentum set to 0.2 , and the sigmoid activation function are used for the training phase.

\subsection{Testing Result and Discussion}

The proposed model was evaluated using the Receiver Operating Characteristic (ROC) to evaluate the accuracy of learning style estimation. The True Positive Rate (TPR) and False Positive Rate (FPR) of the prediction outcomes are used to build a graphical curve called the ROC [28]. Four TPR and FPR values have been estimated for each FSLSM dimension and are shown in Table 4. After training the ANN, optimal values of the weights are stored to be used for testing purposes.

The FPR and TPR are calculated as follows:

$$
\begin{aligned}
F P R & =\frac{\boldsymbol{F P}(\text { False Positive })}{\boldsymbol{F P}(\text { False Positive })+\boldsymbol{T} \boldsymbol{N}(\text { True Negative })} \\
T P R & =\frac{\boldsymbol{T P}(\text { True Positive })}{\boldsymbol{T P}(\text { True Positive })+\boldsymbol{F N}(\text { False negative })}
\end{aligned}
$$

The four ROC curves that describe the prediction accuracy for each dimension are shown in Fig. 2.

Table 4. False Positive Rate (FPR) and True Positive Rate (TPR) values for each FSLM dimensions

\begin{tabular}{|c|c|c|c|c|c|c|c|}
\hline \multicolumn{2}{|c|}{ Perception } & \multicolumn{2}{c|}{ Input } & \multicolumn{2}{c|}{ Processing } & \multicolumn{2}{c|}{ Understanding } \\
\hline $\boldsymbol{F P R}$ & $\boldsymbol{T P R}$ & $\boldsymbol{F P R}$ & $\boldsymbol{T P R}$ & $\boldsymbol{F P R}$ & $\boldsymbol{T P R}$ & $\boldsymbol{F P R}$ & $\boldsymbol{T P R}$ \\
\hline 1.0000 & 1.0000 & 1.0000 & 1.0000 & 1.0000 & 1.0000 & 1.000 & 1.0000 \\
\hline 0.5714 & 0.9241 & 0.5714 & 0.9241 & 0.5714 & 0.9241 & 0.5455 & 0.9775 \\
\hline 0.5238 & 0.8987 & 0.5238 & 0.8987 & 0.5238 & 0.8987 & 0.4545 & 0.9438 \\
\hline 0.4286 & 0.8734 & 0.4286 & 0.8734 & 0.4286 & 0.8734 & 0.1818 & 0.8734 \\
\hline 0.0476 & 0.6835 & 0.0476 & 0.6835 & 0.0476 & 0.6835 & 0.0476 & 0.7640 \\
\hline 0.000 & 0.2911 & 0.000 & 0.2911 & 0.000 & 0.2911 & 0.000 & 0.3820 \\
\hline
\end{tabular}

Students' data are grouped into six separate partitions and research sets of sample splitting ratios of $90 \%$ and $10 \%, 80 \%$ and $20 \%, 70 \%$ and $30 \%, 60 \%$ and $40 \%, 50 \%$ and $50 \%$, and $40 \%$ and $60 \%$. Table 5 displays the measuring precision of the various 
sets' partitions for each dimension of FSLSM. The prediction of $90 \%$ training data and $10 \%$ testing data yields the best precision. The ROC curve clearly shows that the perception, input, and processing measurements are almost the same. Using this splitting ratio, which reflects prediction accuracy, data, and processing is a good fit for the model since they are all above the diagonal line. This means that the proposed model is a strong prediction for student data based on the ROC and will assist facilitators in adapting learning contents based on student learning behavior for inclusive learning experiences.

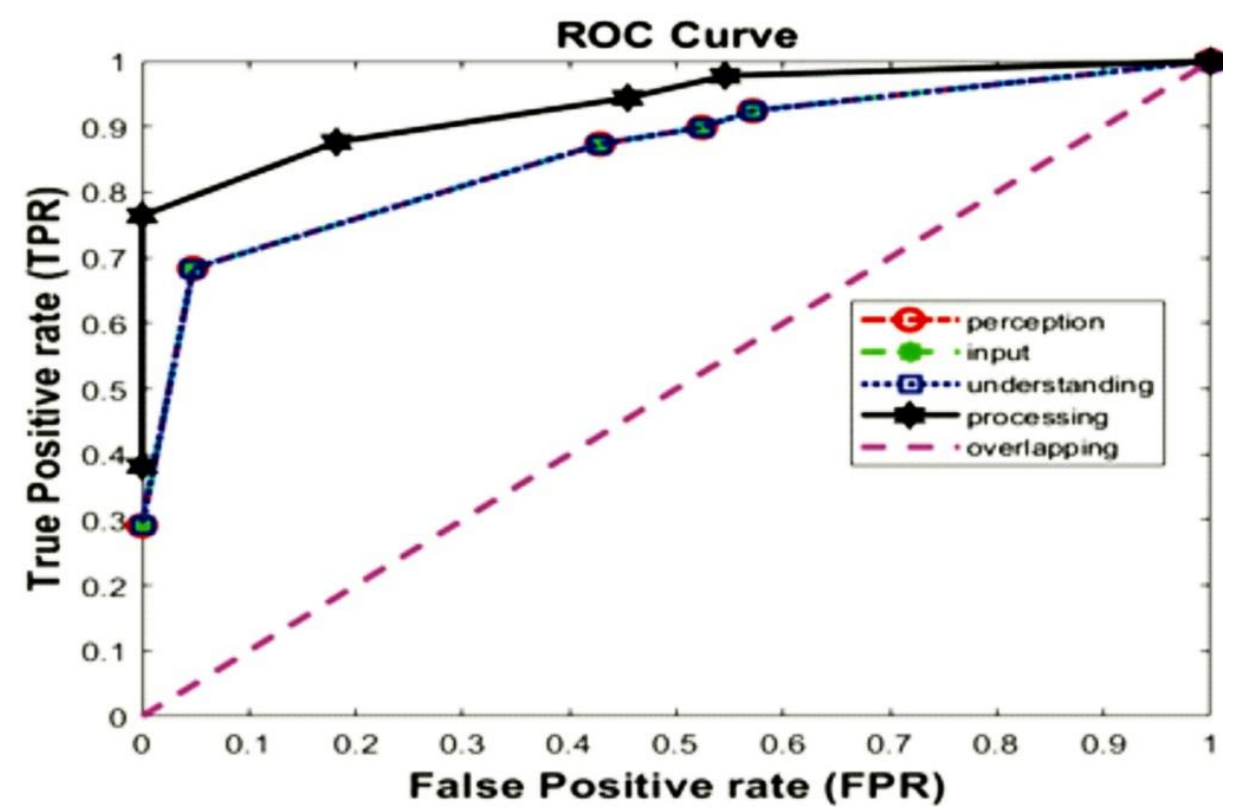

Fig. 2. Four ROC curve of prediction accuracy for each dimension

Table 5. Testing accuracy of classification with the various partition of experiment

\begin{tabular}{|l|c|c|c|c|}
\hline \multirow{2}{*}{$\begin{array}{c}\text { Data Partition } \\
\text { Training and } \\
\text { Testing }\end{array}$} & \multicolumn{4}{|c|}{ Testing Accuracy } \\
\cline { 2 - 5 } & $\begin{array}{c}\text { Perception } \\
\text { Dimension }\end{array}$ & $\begin{array}{c}\text { Input } \\
\text { Dimension }\end{array}$ & $\begin{array}{c}\text { Processing } \\
\text { Dimension }\end{array}$ & $\begin{array}{c}\text { Understanding } \\
\text { Dimension }\end{array}$ \\
\hline $90 \%$ and $10 \%$ & $80 \%$ & $80 \%$ & $80 \%$ & $93 \%$ \\
\hline $80 \%$ and $20 \%$ & $80 \%$ & $80 \%$ & $80 \%$ & $85 \%$ \\
\hline $70 \%$ and $30 \%$ & $78 \%$ & $78 \%$ & $78 \%$ & $94 \%$ \\
\hline $60 \%$ and $40 \%$ & $81 \%$ & $81 \%$ & $81 \%$ & $90 \%$ \\
\hline $50 \%$ and 50\% & $79 \%$ & $79 \%$ & $79 \%$ & $91 \%$ \\
\hline $40 \%$ and $60 \%$ & $78 \%$ & $78 \%$ & $78 \%$ & $90 \%$ \\
\hline
\end{tabular}




\section{Conclusion}

This paper proposes an ANN-based learning agent that classifies students' learning styles based on metacognitive skills using FSLM to support the development of a selfregulated smart learning environment. This approach provided two-fold advantages. First, automatic classification of learning style in a learning environment provides hidden information about a student to facilitate support. Secondly, the method uses recent data about students' actions, which can dynamically recognize students' learning styles to provide further information about the student learning process and support. The method uses metacognitive skills to classify learning styles, and the finding is a promising one compared to the previous works [12-16]. The implication is that metacognitive skills need to develop into a learning environment, which requires restructuring the learning curriculum and pedagogical framework. This work provides a foundation upon which metacognitive skills will be developed into lifelong learning to train students on the skills and knowledge needed to live and contribute to society. While the evaluation was able to provide a good level of classification, however, it is not without limitation. The data used was small and simulated, and there is the possibility that if more data are used, a better result can be obtained. The choice of arbitrary weight is subjective, and there is a possibility that if different values are chosen and normalized, it might give different simulated data sets. These we consider are limitations to this work. We plan to extend our study in the future by expanding the number of data and by deeply analyzing with a variety of data mining algorithms. In addition, for comparison, we can run the test for other common learning styles such as VARK, Kolb, Honey Mumford, and so on. In addition, some cutting-edge machine learning algorithms will be used for comparison. The findings of this study are now being used in the development of a selfregulated smart learning environment to support students learning experiences.

\section{Acknowledgement}

The authors wish to thanks Adamawa State University Mubi, Nigeria, for sponsoring this research work.

\section{$7 \quad$ Availability of Data and Materials}

All data generated or analyzed during this study are attached as supplementary material.

\section{References}

[1] Abdullah, M. A. (2015). Learning style classification based on student's behavior in moodle learning management system. Transactions on Machine Learning and Artificial Intelligence, 3(1), 28. https://doi.org/10.14738/tmlai.31.868 
Paper-An Artificial Neural Network (ANN)-Based Learning Agent for Classifying Learning...

[2] Bakar, Z., A., \& Ali, R. (2016). Learning style constructs in student's learning. Mimbar Pendidikan, 1(2). https://doi.org/10.1108/00400910610651728

[3] Hasibuan, M. S., Nugroho, L. E., \& Santosa, P. I. (2019). Model Detecting Learning Styles with Artificial Neural Network. Journal of Technology and Science Education, 9(1), 85-95. https://doi.org/10.3926/jotse.540

[4] Jalal, A., \& Mahmood, M. (2019). Students' behavior mining in e-learning environment using cognitive processes with information technologies. Education and Information Technologies, 24(5), 2797-2821. https://doi.org/10.1007/s10639-019-09892-5

[5] Troussas, C., Chrysafiadi, K., \& Virvou, M. (2019). An intelligent adaptive fuzzy-based inference system for computer-assisted language learning. Expert Systems with Applications, 127, 85-96. https://doi.org/10.1016/j.eswa.2019.03.003

[6] Coffield, F., Moseley, D., Hall, E., \& Ecclestone, K. (2004). Should we be using learning styles? What research has to say to practice. https://doi.org/10.12691/education-2-9-8

[7] Kolb, D. A., Boyatzis, R. E., \& Mainemelis, C. (2001). Experiential learning theory: Previous research and new directions. Perspectives on thinking, learning, and cognitive styles, 1(8), 227-247. https://doi.org/10.4324/9781410605986-9

[8] Gardner, H. (1998). A Reply to Perry D. Klein's" Multiplying the Problems of Intelligence by Eight." Canadian Journal of Education/Revue canadienne de l'éducation, 23(1), 96-102. https://doi.org/10.2307/1585968

[9] Felder, R. M., \& Silverman, L. K. (1988). Learning and teaching styles in engineering education. Engineering education, 78(7), 674-681.

[10] Biggs, J. B. (1987). Student Approaches to Learning and Studying. Research Monograph. Australian Council for Educational Research Ltd., Radford House, Frederick St., Hawthorn 3122, Australia.

[11] Azzi, I., Jeghal, A., Radouane, A., Yahyaouy, A., \& Tairi, H. (2020). A robust classification to predict learning styles in adaptive e-learning systems. Education and Information Technologies 25(1), 437-448. https://doi.org/10.1007/s10639-019-09956-6

[12] Bajaj, R., \& Sharma, V. (2018). Smart education with artificial intelligence-based determination of learning styles. Procedia computer science, 132, 834-842. https://doi.org/10.1016/j.procs.2018.05.095

[13] Kolekar, S.V., Pai, R.M., \& MM, M.P. (2017). Prediction of learner's profile based on learning styles in an adaptive e-learning system. International Journal of Emerging Technologies in Learning (iJET) 12(6), 31-51. https://doi.org/10.3991/ijet.v12i06.6579

[14] Kose, U. (2013). An Artificial Neural Networks Based Software System for Improved Learning Experience. In 2013 12th International Conference on Machine Learning and Applications (2), 549-554). IEEE. https://doi.org/10.1109/ICMLA.2013.175

[15] Melesko, J., \& Kurilovas, E. (2018). Semantic technologies in e-learning: Learning analytics and artificial neural networks in personalized learning systems. Proceedings of the 8th International Conference on Web Intelligence, Mining and Semantics, 1-7. https://doi.org/10.1145/3227609.3227669

[16] Zakrzewski, A.C., Wisniewski, M.G., Williams, H.L., Berry, J.M. (2020). Artificial neural networks reveal individual differences in the metacognitive monitoring of memory. PloS one 14(7). https://doi.org/10.1371/journal.pone.0220526

[17] Gambo, Y., \& Shakir, M. Z. (2021, March). WIP: Model of Self-Regulated Smart Learning Environment. In 2021 IEEE World Conference on Engineering Education (EDUNINE) (pp. 1-4). IEEE. https://doi.org/10.1109/edunine51952.2021.9429090

[18] Chris B. (2002). Supervised and Unsupervised Land Use Classification. Advanced Image Processing Class at Emporia State University http://academic.emporia.edu/aberjame/student lbanman5/perry3.html 
Paper-An Artificial Neural Network (ANN)-Based Learning Agent for Classifying Learning...

[19] Amatriain, X., Jaimes, A., Oliver, N., \& Pujol, J. M. (2011). Data mining methods for recommender systems. In Recommender systems handbook (pp. 39-71). Springer, Boston, MA. https://doi.org/10.1007/978-0-387-85820-3_2

[20] Csaji, B.C. (2001). Approximation with artificial neural networks (M. Sc Thesis). Faculty of Sciences, Etvs Lornd University, Hungary 24(48). https://doi.org/10.1.1.101.2647

[21] Charaf, H., \& Vajk, I. (1998). A new structure for nonlinear system identification using neural networks. Electrical Engineering and Computer Science 42(2), 175-192.

[22] Minsky, M., \& Papert, S. A. (2017). Perceptron: An introduction to computational geometry. MIT press.

[23] Phansalkar, V. V., \& Sastry, P. S. (1994). Analysis of the back-propagation algorithm with momentum. IEEE Transactions on Neural Networks, 5(3), 505-506. https://doi.org/ $\underline{10.1109 / 72.286925}$

[24] LeCunn, Y. (1998). A theoretical framework for back-propagation. Proceedings of the Connectionist Models Summer schools CMU, Pittsburgh, (1), 234-268

[25] Rosenblatt, F. (1957). The perceptron, a perceiving and recognizing automaton (Project Para Report No. 85-460-1). Ithaca, NY: Cornell Aeronautical Laboratory (CAL).

[26] Rumelhart, D. E., Hinton, G. E., \& Williams, R. J. (1986). Learning representations by backpropagating errors. nature, 323(6088), 533-536. https://doi.org/10.1038/323533a0

[27] El-Bishouty, M. M., Aldraiweesh, A., Alturki, U., Tortorella, R., Yang, J., Chang, T. W., \& Graf, S. (2019). Use of Felder and Silverman learning style model for online course design. Educational Technology Research and Development, 67(1), 161-177. https://doi.org/ 10.1007/s11423-018-9634-6

[28] Tom F. (2006). An introduction to ROC analysis. Pattern Recognition Letters 27, 861-874.

\section{Authors}

Yusufu Gambo is currently a $\mathrm{PhD}$ candidate at the School: Computing, Engineering and Physical Sciences, University of the West of Scotland (UWS), UK. He received BTech in Operations Research from Federal University of Technology, Yola, Nigeria and MSc in Information Technology from Stirling University, Scotland, UK. He is a lecturer at the Department of Computer Science, Adamawa State University, Mubi, Nigeria. He is a member of IEEE and has some publications in both peer review journals and conferences.

Muhammad Zeeshan Shakir is Reader (Associate Professor) in Computer Networks at the University of the West of Scotland (UWS), UK, received over $£ 3 \mathrm{~m}$ research funding from bodies such as Innovate UK, ERASMUS, QNRF and UK industries. With over 10 years of research expertise in design and development of information and communication technologies (ICT), he has published over 150 research articles and edited or contributed to 10 books. He has an interest in wireless technologies and application of tools such as Artificial Intelligence \& Machine Learning to support global connectivity around the world and related emerging applications. He is a recipient of IEEE Communications Society Fred W. Ellersick Award 2021, IEEE Communications Society and China Institute of Communications Best Journal Article Award 2019 and UWS STARS Award 2020 \& 2018 (Staff Appreciation and Recognition scheme) for outstanding research and enterprise performance. He has been serving as a Chair and organising committee of several symposiums/workshops in IEEE flagship 
Paper-An Artificial Neural Network (ANN)-Based Learning Agent for Classifying Learning...

conferences, including Globecom, ICC and WCNC. He is an Editor of PHYCOM, IEEE Communications Letters and served as a guest editor to IEEE Wireless Communications, IEEE Communications, and IEEE Access. He is a founding Chair of IEEE ComSoc emerging technologies committee on backhaul/fronthaul. He is a Fellow of Higher Education Academy, UK, Senior Member of IEEE, and an active member of IEEE ComSoc and member of International Association of Smart Learning Environments (IASLE). Recently, he has been selected (through a competitive process) by the Scottish Parliament as a Member Scottish Parliament Framework Agreement Covid-19 Pandemic to provide expert advice on the impact of Covid-19 on digital infrastructure, energy, education, and healthcare. (Email: muhammad.shakir@uws.ac.uk).

Article submitted 2021-05-25. Resubmitted 2021-07-03. Final acceptance 2021-07-07. Final version published as submitted by the authors. 